\title{
Necrotizing Sialometaplasia of Palate
}

\section{Radka Cholakova*}

\author{
*Department of Oral Surgery, Faculty of Dental Medicine, Medical University -Plovdiv, Bulgaria \\ *Corresponding author: Radka Cholakova, Department of Oral Surgery, Faculty of Dental Medicine, Medical University - Plo- \\ vdiv, Bulgaria.
}

\section{Abstract}

Necrotizing sialometaplasia (NS) is a rare, benign, inflammatory condition, occasionally with ulcers, which is self-limiting and affects mainly salivary tissue.

Purpose: To present a case of NS associated with a systemic connective tissue disease in a female patient.

Material/Methods: A 56-year-old female patient with ulcers of the palate, surrounded by a red halo, for 2 weeks, which did not resolve when treated with topical antiseptics. Clinical and radiological methods, together with pathohistological analysis, were used to make the diagnosis. Immunohistochemical analyses to diagnose patient's systemic disease were performed at a rheumatology clinic.

Results: The definitive diagnosis was made based on a pathological examination and the tests performed in the rheumatology clinic, which found that this was a case of necrotizing sialometaplasia of the minor salivary glands and a concomitant systemic connective tissue disease.

Conclusions: NS is a rare disease, with an excellent prognosis, without any possible preventive strategies.

Keywords : Necrotizing sialometaplasia; diseases of the minor salivary glands; tumour-like lesion

\section{Introduction}

Necrotizing sialometaplasia (NS) is a rare, benign, inflammatory condition, sometimes with ulcers, which is self-limiting and affects mainly salivary tissue. This disease is classified as a "tumour-like lesion" in the WHO classification of salivary gland tumours. It was first described in 1973 by Abrams, Melrose and Howell [1], and in the following year, Dunlap and Barker reported five diagnosed cases [2]. This lesion can be confused with a malignant disease, thus resulting in unnecessary radical surgery.

\section{Materials and Methods}

A female patient of visible age of 56 , corresponding to her calendar age, with complaints of a non-healing "aphthous ulcer", which appeared 2 weeks earlier. Treatment with antiseptic mouthwashes and topical application of propolis was administered. The aphthous ulcer did not resolve, but the pain subsided over time. The initial examination showed two ulcers with dimensions of about $2 \mathrm{~mm}$, located on either side of the median palatine suture, with a red halo around them. After treatment with SOLCOSERYL (MEDA Pharma GmbH \& Co. KG, Germany), the ulcers resolved, but the red spots remained (Figure 1). The patient was referred for a CBCT in order to detect erosions in the palatine bone under the ulcers (Figure 2). The size of the formation did not decrease and, therefore, an excisional biopsy was performed in full thickness of one of the lesions, and then was provided for histopathological examination. The wound was covered with a PRF membrane and healed without complications. Pathological examination showed that there were cords of the lining multilayered squamous nonkeratinizing epithelium, deep below it, without atypicality, and with rapid proliferation of granulation tissue, which covered the minor salivary gland ducts, with squamous cell metaplasia. The patient was a moderate smoker (up to 10 cigarettes/day). She had cholelithiasis without clinical manifestations. She had had surgery for a benign neoplasm of the breast several years before. Intermittent complaints of swelling and rash on the upper eyelids, erythematous rash on the nasal dorsum and photosensitivity were 
Figure 1: NS of the palate, without ulcers. The lesions are located on both sides of the palate.

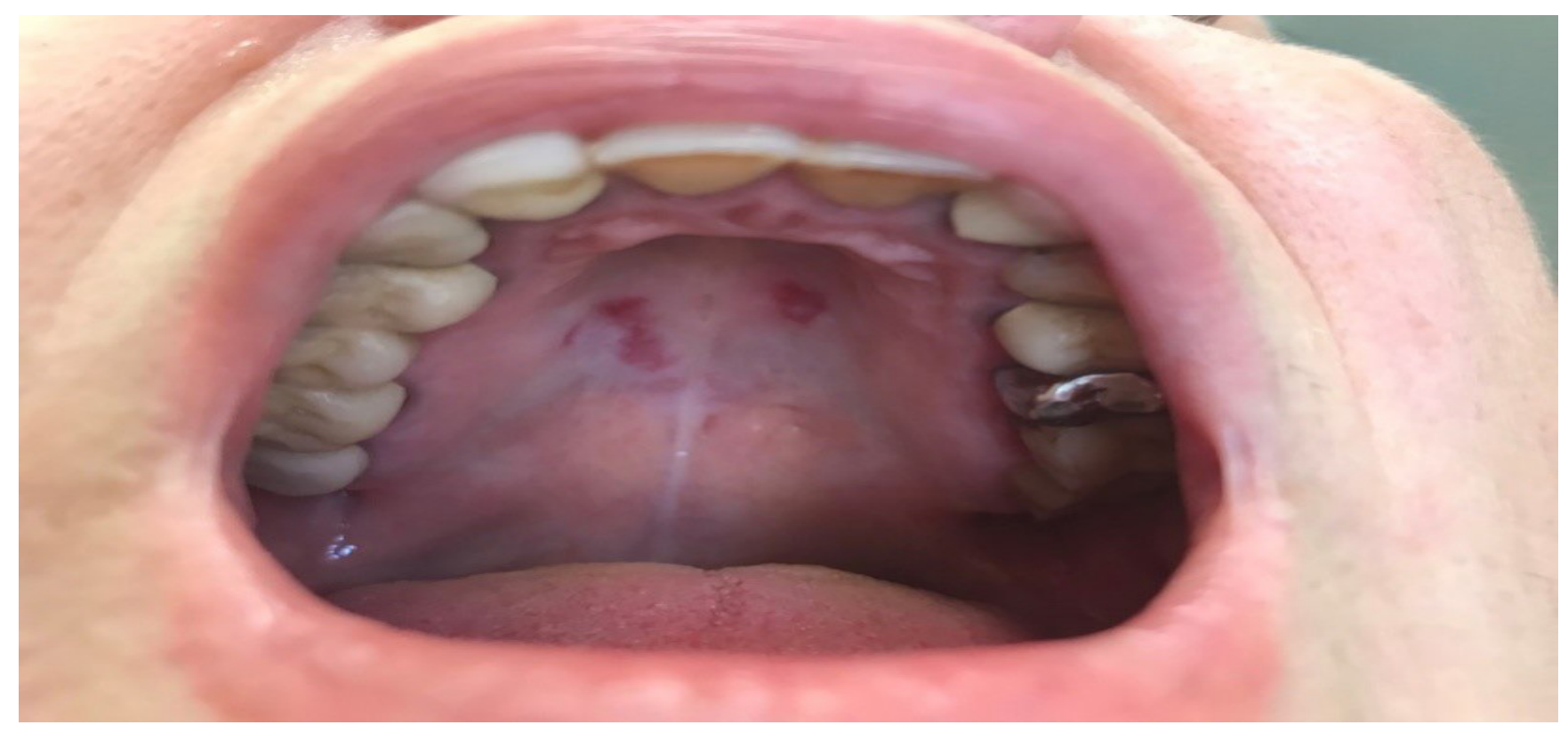

Figure 2: CBCT scan of the maxilla, showing no bone involvement.

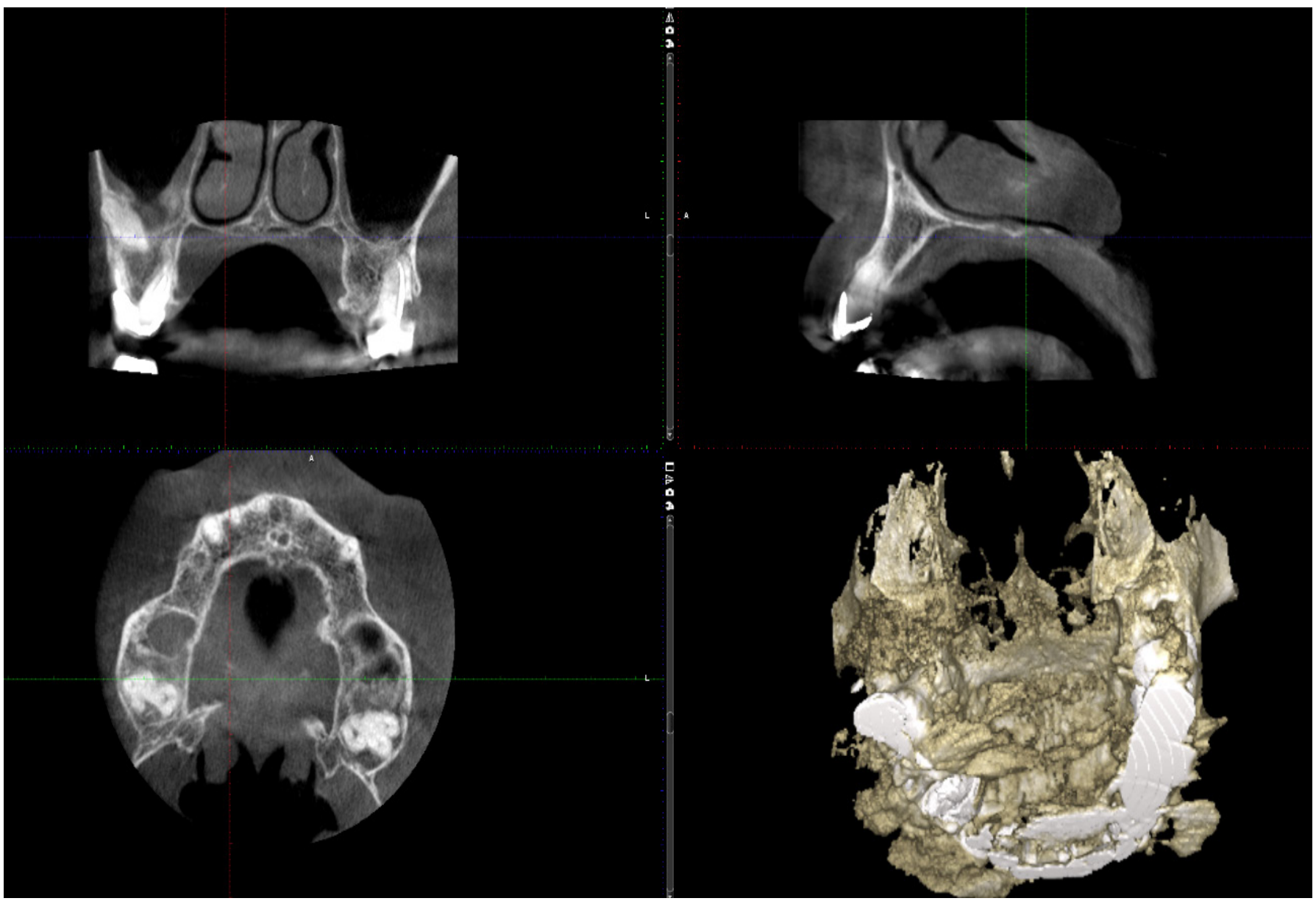

Citation: Radka Cholakova* . Necrotizing Sialometaplasia of Palate.Op Acc J Bio Sci \& Res 6(4)-2020. 
present, for which the patient had been admitted to a rheumatology clinic for examination. A systemic connective tissue disease was suspected there, as elevated total AHA, Anti-SS-A and Anti-SSB antibodies with very high titers and low C4 complement level, without significant proteinuria, were found. The dermatological examination showed pathological skin lesions of the nose with erythematousedematous plaque, $6 \mathrm{~cm}$ in diameter. Sjögren's syndrome or systemic lupus erythematosus were suspected in this patient. The pathohistological examination of the minor salivary glands from the lower lip mucosa found that this was not a case of Sjögren's syndrome. Biopsy of the plaque of the nasal lesion was recommended.

\section{Results}

The definitive diagnosis was made based on a pathological examination and the tests performed in the rheumatology clinic, which found that this was a case of necrotizing sialometaplasia of the minor salivary glands and a concomitant systemic connective tissue disease.

\section{Discussion}

Necrotizing sialometaplasia is extremely rare, accounting for less than $1 \%$ of oral lesion biopsies [3]. The mean age of onset is 46 years, and the male:female ratio is 2.7:1 [4]. The disease is prevalent among the Caucasian race, with Caucasian:African American ratio of $5: 1$, according to Brannon [5]. In most cases (80\%), the minor palatine salivary glands are affected. Although rarely, it can occur in the retromolar space, gingiva, lips, tongue, cheeks, and nasal cavity $[1,6]$. This disease can also affect the large salivary glands in more than $10 \%$ of the cases [3]. The etiopathogenesis of necrotizing sialometaplasia is unknown, but it is thought that the lesion develops as a result of previous ischemia in the salivary gland. In experimental models, disruption of the arterial blood supply to the salivary glands in rodents results in a NS-like histopathological picture. This disease is found in patients with sickle cell disease, Buerger's disease and Raynaud's phenomenon, which are all vasculopathies that predispose to ischemia. Other risk factors for the development of NS include: smoking (and alcohol consumption), use of cocaine and anabolic steroids, hot food, fellatio, traumatic vascular injury, and bulimia [7-9]. The synergistic action of NSAIDs and alcohol over a long period of time results in a change in the oral mucosa functions due to suppression of prostaglandin production and reduction in the blood supply to the minor salivary glands, which causes ischemic events [10]. The iatrogenic factors for the development of NS are the use of local infiltration anesthesia with an anesthetic with a higher concentration of correctives, intubation, bronchoscopy, local radiotherapy, as well as surgical procedures in the vicinity of the affected area $[3,7,11,12]$, which, in the case described by us, had not been conducted for a period of more than 6 months. Senapati et al. [13] reported that NS is a manifestation of local vasculitis. It may be associated with other tumours, in particular Warthin's tumour, Abrisokov's tumour, lip cancer, rapidly growing malignant mesenchymal disease and salivary gland tumours. There is also a connection with previous upper respiratory tract diseases (chronic sinusitis and allergies) in the past few weeks. It is possible that ischemia is due to immune complexes, resembling the pathogenesis of erythema multiforme or benign trigeminal sensory neuropathy. In our case, levels of immune complexes in the body were elevated.

Anneroth and Hansen described the following five clinical stages in the development of necrotizing sialometaplasia: infarction, sequestration, ulceration, repair and healing [6]. A subacute variant of this condition was also described in the literature. Histological features are ischemic lobular necrosis of seromucinous glands, with maintenance of intact lobular architecture, despite coagulative necrosis of the mucinous acini. Pale acinar outlines often persist, but the cell nuclei are hypochromatic or absent. Mucin extravasation into the adjacent tissues triggers an inflammatory reaction dominated by histiocytes and granulation tissue. Within the necrotic lobules, the inflammatory component is often minimal, but is usually found in the surrounding tissues. Although squamous metaplasia of ducts and acini is typical (which makes the diagnosis challenging due to its similarity to malignancies), the metaplastic cells have benign nuclear morphology, with minimal pleomorphism or hyperchromatism and few mitotic figures. Nests of squamous epithelium, which usually have smooth contours, occasionally may have an irregular outline.

Pseudoepitheliomatous hyperplasia, where the overlying or adjacent epithelium is markedly hyperplastic, together with extensive ductal metaplasia, may resemble malignant condition of the epithelium, which could be the reason for misdiagnosis and radical ablative surgery. It may be difficult to distinguish NS from squamous cell carcinoma, low-grade mucoepidermoid carcinoma and oncocytic tumours. Specific histopathological characteristics may have some relation to the "age" of the lesion at biopsy. Coagulative necrosis is more common in "new" lesions, whereas fibrosis and squamous metaplasia are typical of "older" lesions. In our case, biopsy was taken nearly 2 weeks after the onset of the first symptoms, and therefore the changes correspond to an "old" lesion. Management of this disease includes monitoring, use of topical antiseptics, 
and pain control until recovery [3]. In the presence of predisposing factors, their correction is necessary.

\section{Conclusion}

The prognosis of NS is excellent, provided that the correct diagnosis is made. No preventative strategies are known.

\section{References}

1. Abrams AM, Melrose RJ, Howell FV (1973) Necrotizing sialometaplasia. A disease simulating malignancy. Cancer 32(1): 130-135.

2. Dunlap CL, Barker BF (1974) Necrotizing sialometaplasia: report of five additional cases. Oral Surg 37(5): 722-727.

3. Balaji SM, Balaji P (2015) Surgical management of necrotizing sialometaplasia of palate. Indian J Dent Res 26(5): 550-555.

4. Lynch DP, Crago CA, Martinez MG Jr (1979) Necrotizing sialometaplasia. A review of the literature and report of two additional cases. Oral Surg Oral Med Oral Pathol 47(1): 63-69.

5. Brannon RB, Fowler CB, Hartman KS (1991) Necrotizing sialometaplasia. A clinicopathologic study of sixty-nine cases and review of the literature. Oral Surg Oral Med Oral Pathol 72(3): 317-325.
6. Anneroth G, Hansen LS (1982) Necrotizing sialometaplasia. The relationship of its pathogenesis to its clinical characteristics. Int J Oral Surg 11(5): 283-291.

7. Schöning H, Emshoff R, Kreczy A (1998) Necrotizing sialometaplasia in two patients with bulimia and chronic vomiting. Int J Oral Maxillofac Surg 27(6): 463-465.

8. Abdalla-Aslan R, Frid H, Totri A, Akrish S, Merhav G, et al. (2020) Necrotizing sialometaplasia of the palate in a young bodybuilder with anabolic androgenic steroids abuse. Quintessence Int 51(6): 496-501.

9. Salvado F, Nobre MA, Gomes J, Maia P (2020) Necrotizing Sialometaplasia and Bulimia: A Case Report. Medicina (Kaunas). 56(4): 188.

10. Gatti A, Broccardo E, Poglio G, Benech A (2016) Necrotizing Sialometaplasia of the Hard Palate in a Patient Treated with Topical Nonsteroidal Anti-Inflammatory Drug. Case Rep Dent 2016: 9545861.

11. Daudia A, Murty GE (2002) First case of full-thickness palatal necrotizing sialometaplasia. J Laryngol Otol. 116(3): 219-220.

12. Farina D, Gavazzi E, Avigo C, Borghesi A, Maroldi R (2008) Case report. MRI findings of necrotizing sialometaplasia. Br J Radiol 81(966): e173-5.

13. Senapati S, Samal SC, Kumar R, Patra S (2016) Necrotizing sialometaplasia: Manifestation of a localized unclassified vasculitis. Indian J Pathol Microbiol 59(2): 232-234.

*Corresponding author: Radka Cholakova, Email: r_cholakova1978@abv.bg

Next Submission with BGSR follows:

- Rapid Peer Review

- Reprints for Original Copy

- E-Prints Availability

- Below URL for auxiliary Submission Link: https://biogenericpublishers.com/submit-manuscript/ 